\title{
Investigation of QT Prolongation with Hydroxychloroquine and Azithromycin for the Treatment of COVID-19
}

\author{
Avni Uygar Seyhan ${ }^{1}$, Fatih Doganay ${ }^{2}$, Erdal Yilmaz ${ }^{1}$, Nurdan Papila Topal ${ }^{3}$ and Rohat Ak $^{1}$ \\ ${ }^{1}$ Department of Emergency Medicine, Kartal Dr. Lutfi Kirdar City Hospital, Turkey \\ ${ }^{2}$ Department of Emergency Medicine, Edremit State Hospital, Turkey \\ ${ }^{3}$ Department of Cardiology, Kartal Dr. Lutfi Kirdar City Hospital, Turkey
}

\begin{abstract}
Objective: To assess and identify the risk of prolonged QT about hydroxychloroquine (HQ) and azithromycin (AZ) used in the treatment of patients with COVID-19.

Study Design: Cohort study.

Place and Duration of Study: Kartal Dr. Lütfi Kırdar City Hospital, İstanbul, Turkey, from March to May 2020.

Methodology: One hundred and forty-four patients with the diagnosis of COVID-19, confirmed by Rt-PCR (reverse transcription-polymerase chain reaction), were restrospectively reviewed. Patients who were hospitalised, received $\mathrm{HQ}$ or $\mathrm{HQ}$ plus $\mathrm{AZ}$ treatment, had a baseline electrocardiogram (ECG), and had at least one ECG after treatment were included in the study. Patients with missing data were excluded.

Results: Fifty-one (35.4\%) patients were given hydroxychloroquine monoterapy (HQ), 93 (64.6\%) were given hydroxychloroquine plus azithromycin (HA), and $70(48.6 \%)$ were women. Pre-treatment mean QTc measurements were calculated as $410.61 \pm$ 29.44 milliseconds (ms) for HQ group and $412.02 \pm 25.37 \mathrm{~ms}$ for HA group, while the mean values of post-treatment QTc measurements were calculated as $432.31 \pm 33.97 \mathrm{~ms}$ for HQ group and $432.03 \pm 27.0$ ms for the HA group. Post-treatment QTC measurements of both HA group and HQ group were prolonged compared to pre-treatment measurements. Ventricular arrhythmia was not observed in any patient.

Conclusion: For COVID-19, no globally accepted definite treatment has yet been found. Both of hydroxychloroquine monotherapy and hydroxychloroquine plus azithromycin treatment regimens cause QTc measurement to increase at a statistically significant level. We concluded that this increase in QTc did not cause ventricular arrhythmia.
\end{abstract}

Key Words: COVID-19, QTC interval, Hydroxychloroquine, Azithromycin.

How to cite this article: Seyhan AU, Doganay F, Yilmaz E, Topal NP, Ak R. Investigation of QT Prolongation with Hydroxychloroquine and Azithromycin for the Treatment of COVID-19. J Coll Physicians Surg Pak 2020; 30(JCPSPCR):CR153-CR157.

\section{INTRODUCTION}

In December 2019, an unexplained pneumonia outbreak occurred in Wuhan, and it was reported that the cause of this outbreak is a new coronavirus infection called COVID-19 (Corona Virus Disease 2019). ${ }^{1}$ As on 21 October, the virus caused more than 40 million cases and more than one million deaths across the world; and according to the data of the Ministry of Health, more than 350,000 cases and more than 9,000 deaths were seen in Turkey. ${ }^{2,3}$

While new drug-vaccination studies are underway to treat the disease, available drugs were also tried for treatment.

Correspondence to: Dr. Rohat Ak, Department of Emergency Medicine, Kartal Dr. Lutfi Kirdar City Hospital, Turkey

E-mail: rohatakmd@gmail.com

Received: August 18, 2020; Revised: October 22, 2020;

Accepted: November 04, 2020

DOI: https://doi.org/10.29271/jcpsp.2020.JCPSPCR.CR153
Hydroxychloroquine has a strong antiviral activity in vitro, and it was reported that it inhibits SARS-CoV-2 and gives better results when used with azithromycin. ${ }^{4,5}$ Subsequent studies suggest that this combination had no clinical benefit. ${ }^{6,7}$ Furthermore, both azithromycin and hydroxychloroquine are associated with prolonged QTc, and combined use is likely to strengthen this negative effect. ${ }^{7}$ Hydroxychloroquine prolongs the QT by blocking the activation of the potassium channel IKr (hERG / Kv11.1). ${ }^{8}$ Azithromycin prolongs the QT interval due to the prolongation of the action potential. ${ }^{9}$

The aim of this study was to assess and identify the risk of prolonged QTC about the use of accompanying hydroxychloroquine or the use of hydroxychloroquine plus azithromycin.

\section{METHODOLOGY}

The study was included patients who had received in-patient treatment at Kartal Dr. Lütfi Kırdar City Hospital, İstanbul, Turkey, between March 11 and May 11, 2020, with COVID-19 diagnosis confirmed via Rt-PCR test, and who were administered hydroxychloroquine or hydroxychloroquine plus 
azithromycin with baseline 12 lead electrocardiogram (ECG) and at least one ECG after drug administration. Data of these patients was accessed by searching the electronic patient registration system of the hospital. Patients who were hospitalised, received $\mathrm{HQ}$ or $\mathrm{HQ}$ plus $\mathrm{AZ}$ treatment, had a baseline electrocardiogram (ECG), and had at least 1 ECG after treatment were included in the study. Patients with missing data were excluded.

To standardise QT length according to the heart rates of the patients, ECG records were examined by a cardiologist and Bazett formula (QTc $=\mathrm{QT} / \sqrt{ } \mathrm{RR}$ ) was used for those with heart rate within the range of $60-100 / \mathrm{min}$, and Framingham formula $(\mathrm{QTC}=\mathrm{QT}+0.154(1-\mathrm{RR}))$ was used for those with heart rate outside the range of $60-100 / \mathrm{min}$. Prolonged QTc was defined as an increase of more than 60 milliseconds $(\Delta Q T c>60$ milliseconds) in QTc intervals compared to the beginning or a QTc of 500 milliseconds or above. ${ }^{10}$ Patients were divided into two groups, namely the patients diagnosed with COVID-19 who used hydroxychloroquine only and patients diagnosed with COVID-19 who used hydroxychloroquine together with azithromycin, and statistical analysis was performed. Standard hydroxychloroquine regimen was $400 \mathrm{mg}$ twice in the day one, then 400 mg per day in the days two to five. Azithromycin was administered $250 \mathrm{mg}$ per day for five days.

This study was approved by the Corporate Ethical Committee with a waiver of informed consent due to the retrospective nature of the work.

Analysis of data was performed using the IBM SPSS Statistics 26. The value of $p<0.05$ was considered statistically significant. Categorical data were expressed as frequency and percentage while the parameters having continuous variation as mean \pm S.D.

After the data was collected, two groups were subjected to power analysis via G-power software version 3.1.9.7 using the independent sample t-test and post-hoc options. The power value was calculated as 0.89 taking the effect size $(d)$ as 0.5 and $\alpha$ (error) as 0.05 . Independent sample t-test and a paired sample t-test were performed to compare the effect of treatments on QTcinterval.

To use independent sample t-test and paired sample t-test methods, which are parametric tests, the assumptions of the normality of distributions and the homogeneity of variances were examined.

Kolmogorov-Smirnov normality test was performed to determine the normality of the distributions. It was seen that the normality assumption was achieved. Levene's test was performed to determine whether the variants, another assumption of independent sample t-test, were homogeneous. Levene's test showed for homogeneity of variances of pretreatment $(p=0.127)$ and post-treatment $(p=0.011)$ QTc measurements of the patients included in the study. The hypothesis established for the homogeneity of the distributions of pretreatment QTC measurements was accepted. The homogeneity of post-treatment QTc measurements was refused. Therefore, p- values used in the case of uneven homogeneity during the analysis for post-treatment measurements were reported.

\section{RESULTS}

This study included 144 patients, with 51 (35.4\%) patients in the hydroxychloroquine (HQ) group and 93 (64.6\%) in the hydroxychloroquine plus azithromycin (HA) group. Of these patients, 13 (9\%) were treated in the intensive care unit. Seventy $(48.6 \%)$ patients were females and $74(51.4 \%)$ were males. The age ranged from 13 to 101 years. The average age of all patients included in the study was $55.81 \pm 19.32$ years.

Pre-treatment QTc measurements were calculated as $410.61 \pm$ 29.44 milliseconds (ms) for the HQ group and $412.02 \pm 25.37$ ms for the HA group, while the mean values of post-treatment QTc measurements were calculated as $432.31 \pm 33.97$ ms for the HQ group and $432.03 \pm 27.0 \mathrm{~ms}$ for the HA group. Two patients were dedected with QTc duration over $500 \mathrm{~ms}$ ( 1 in HQ, 1 in HA) for post-treatment QTc measurement. The post-treatment QTc value of three patients increased more than $60 \mathrm{~ms}$ compared to the pre-treatment QTc value ( 1 in HQ, 2 in HA). The patients whose QTc duration over $500 \mathrm{~ms}$ and the patients with QTc prolongation over 60 ms were not same patients.

To determine the source of the difference, independent sample t-test was performed to compare the pre-treatment and posttreatment QTc measurements according to the HQ and HA groups, and paired samplet-test was performed to compare the pre-treatment and post-treatment QTc measurements of the groups. The findings were given in Tablel.

According to the findings, there is no statistically significant difference between pre-treatment QTc measurements of the patients in HQ and HA groups ( $p=0.763$, Table I). Similarly, no statistically significant difference was found between posttreatment QTc measurements of the patients included in the HQ and HA groups ( $p=0.959$, Table I). This finding shows that the increase in post-treatment QTc measurements compared to pre-treatment QTC measurements is not statistically significantly different among the groups (Tablel).

As shown in Table 1, there is a statistically significant difference between pre-treatment and post-treatment QTC measurements of the participants in the HQ group $(p<0.001$, Table I). This difference is in favor of post-treatment measurements. Similarly, the pretreatment and post-treatment QTC measurements of the participants in the HA group varied statistically significantly $(p<0.001$, Table I). This difference is in favor of post-treatmentmeasurements.

\section{DISCUSSION}

Since there is no specific and standard treatment, the treatment of COVID-19 patients poses a serious problem worldwide. The prolonged QTc and causing ventricular arrhythmias such as Torsades de Pointes, as reported in the literature for hydroxychloroquine and azithromycin which are currently in use, cause concern among physicians in terms of patient management. $^{11,12}$ 
Table I: Sample table for Independent sample and paired sample T-test.

Table I: Sample table for Independent sample and paired sample T-test.
\begin{tabular}{|l|c|c|c|}
\hline Measurements & $\begin{array}{c}\text { HQ Group (n=51) } \\
\text { (Mean } \pm \text { S.D.) }\end{array}$ & $\begin{array}{c}\text { HA Group (n=93) } \\
\text { (Mean } \pm \text { S.D.) }\end{array}$ & $\begin{array}{c}\text { All Patiens (n=144) } \\
\text { (Mean } \pm \text { S.D.) }\end{array}$ \\
\hline Pre-treatment QTC & $410.61 \pm 29.44$ & $412.02 \pm 25.37$ & $411.52 \pm 26.79$ \\
\hline Post-treatment QTC & $432.31 \pm 33.97$ & $432.03 \pm 27.0$ & $432.13 \pm 29.54$ \\
\hline p-values & $<0.001$ & $<0.001$ & 0.959 \\
\hline
\end{tabular}

In this study, when the two groups are compared, there is no significant difference between the pre-treatment QTC measurements. We can say that both groups are similar in terms of pre-treatment QTC measurements.

When the groups are compared in terms of pre-test and post-test, there is a significant difference between pre-treatment and post-treatment QTc measurements in both HQ and HA groups. For both groups, this difference appears to be prolonged in post-treatment QTc measurements. Post-treatment QTc measurements of both HA group and HQ group were prolonged compared to pre-treatment measurements. In this cohort, the QTc times of patients who received hydroxychloroquine (HQ) and hydroxychloroquine plus azithromycin (HA) were prolonged compared to the baseline values, but there were no ventricular arrhythmias that could lead to the end of treatment in patients.

Hydroxychloroquine was first approved by the U.S. Food and Drug Administration in 1955 and generally found to be safe and well-tolerated in patients treated for chronic inflammatory conditions. ${ }^{13}$ It is known that this drug, which is one of the preferred treatment regimens for COVID-19, can block Kv11.1 (HERG) and prolong QT interval. ${ }^{14}$ Clinical arrhythmic toxicity is usually associated with chronic use, use of accompanying QT-prolonging agents (e.g., azithromycin), metabolic irregularities (e.g., hypokalemia), kidney failure, or acute overdose. ${ }^{15}$

The guidelines of the American Heart Association ( $\mathrm{AHA}$ ) suggest that the likelihood of clinical arrhythmic toxicity is very low since the duration of $\mathrm{HQ}$ administration during the COVID-19 treatment process is relatively short (5-10 days). Nevertheless, it is said that it should be used carefully in patients with known congenital prolonged QT syndrome and patients additionally taking QT-prolonging drugs, and the dose should be reduced in patients with severe renal failure, and the patients with electrolyte imbalance can take this drug subject to regular monitoring only after the electrolyte irregularity is treated. ${ }^{16}$

During the COVID-19 pandemic, the effects of HQ and HA treatments on QTC attracted attention and several studies were carried out in this matter. In their retrospective research, Bessière et al. reported 40 patients who received hydroxychloroquine alone in the intensive care unit (400 mg/day for 10 days; $45 \%$ ) or hydroxychloroquine plus azithromycin (250 mg/day for 5 days; 55\%). In this cohort, the baseline QTC was not prolonged (median, 414 milliseconds). It was reported that only $5 \%$ of the patients who took hydroxychloroquine and 33\% of the patients who took both drugs had QTC $\geq 500$ milliseconds and no patient developed arrhythmia. ${ }^{11}$

In their retrospective-observational study, Mercuro et al. examined hydroxychloroquine and QT relationship in 90 hospitalised COVID-19 patients. 53 of the 90 patients who received hydroxychloroquine were simultaneously administered azithromycin, and QTc was measured before and after hydroxychloroquine administration. Baseline median QTC was found longer than usual (only hydroxychloroquine group: 472 milliseconds; hydroxychloroquine plus azithromycin group: 442 milliseconds). Seven patients who took only hydroxychloroquine ( $19 \%$ ) had QTc $\geq 500$ milliseconds, and $21 \%$ of patients who received combination therapy had QTC $\geq 500$ milliseconds. Torsades de pointes was seen in a patient with multiple cardiac and respiratory complications. ${ }^{12}$

In their study, Mazzanti et al. examined the QT relationship of 150 patients who received hydroxychloroquine monotherapy and hydroxychloroquine plus azithromycin and/or lopinavir/ritonavir. Only $2 \%$ of the patients had severe QTc prolongation ( $\geq 500$ milliseconds) and none of the patients had a life-threatening arrhythmia. ${ }^{17}$ In the present, only two patients had severe QT prolongation ( $\geq 500$ milliseconds), one of which was a patient who received hydroxychloroquine monotherapy, while the other was a patient who received hydroxychloroquine plus azithromycin.

In a retrospective two-centered study, Chorin et al. reported that $23 \%$ of the 251 patients who received hydroxychloroquine plus azithromycin combination had QTC $\geq 500$ milliseconds and one patient developed documented ventricular arrhythmia. $^{18}$

In a prospective study, Kuate et al. did not find any significant change in QT time during follow-up of 51 ambulant patients treated with hydroxychloroquine plus azithromycin and no patients had symptomatic arrhythmia during treatment. ${ }^{19}$

In the present study, no patients developed ventricular arrhythmia. It is not clear that the cause of ventricular arrhythmia in the two patients reported in the literature, ${ }^{13,18}$ is due to the incriminated agent use only.

In retrospective studies, it is not possible to perform applications within a specific plan. In this retrospective study, which 
was conducted considering the applications as registered, the data should be evaluated optimally by the nature of retrospective work, although it was tried to collect the data with minimal error. All patients could not be examined during the study due to the missing patient data in the patients' digital files. Since, for some patients, ECG was recorded before the termination of treatment, one cannot rule out that one have missed the maximum effect of the drug on the QTc range. For COVID-19, no globally accepted definite treatment has yet been found. Since hydroxychloroquine and azithromycin which are included in the treatment regimen cause prolonged QTC, these patients need to be monitored carefully. This situation will be further clarified by the wide-scale and prospective studies to be conducted in the future.

\section{CONCLUSION}

Both hydroxychloroquine and hydroxychloroquine plus azithromycin treatment regimens led to a statistically significant increase in QTc measurements. But these elongations were of similar magnitude. That is, the effects of both treatment regimens on QTc prolongation were similar.

\section{ETHICAL APPROVAL:}

This study was conducted in compliance with the ethical principles according to the Declaration of Helsinki, and it was approved by the local Institutional Review Board (Number: 2020/514/178/5).

\section{PATIENTS' CONSENT:}

Since it was designed as a retrospective study, the data were collected from the hospital archive after approval of the Ethics Committee.

\section{CONFLICT OF INTEREST:}

The authors declared no conflict of interest.

\section{AUTHORS' CONTRIBUTION:}

AUS: Substantial contributions to the conception or design of the work.

FD, RA: Drafting the work or revising it critically for important intellectual contents.

EY, NPT: Acquisition, analysis. Interpretation of data for the work.

\section{REFERENCES}

1. Chen N, Zhou M, Dong X, Qu J, Gong F, Han Y, et al. Epidemiological and clinical characteristics of 99 cases of 2019 novel coronavirus pneumonia in Wuhan, China: A descriptive study. Lancet 2020; 395(10223):507-13. doi:10.1016/S0140-6736(20)30211-7.

2. http://covid19.who.int/

3. http://covid19.saglik.gov.tr/

4. Wang M, Cao R, Zhang L, Yang X, Liu J, Xu M, et al. Remdesivirand chloroquine effectively inhibit the recently emerged novel coronavirus (2019-nCoV) in vitro. Cell Res 2020;
30(3):269-271. doi:10.1038/s41422- 020-0282-0.

5. Gautret P, Lagier JC, Parola P, Hoang VT, Meddeb L, Mailhe $M$, et al. Hydroxychloroquine and azithromycin as a treatment of COVID-19: Results of an open-label non-randomised clinical trial. Int J Antimicrob Agents 2020; 56(1):105949. doi:10.1016/j. ijantimicag.2020.105949.

6. Molina JM, Delaugerre C, Le Goff J, Mela-Lima B, Ponscarme $D$, Goldwirt L, et al. No evidence of rapid antiviral clearance or clinical benefit with the combination of hydroxy-chloroquine and azithromycin in patients with severe COVID-19 infection. Med Mal Infect 2020; 50(384): 30085-8. doi: 10.1016/j.medmal.2020.03.006.

7. Rosenberg ES, Dufort EM, Udo T, Wilberschied LA, Kumar J, Tesoriero J, et al. Association of treatment with hydroxychloroquine or azithromycin with in-hospital mortality in patients with COVID-19 in New York state. Jama 2020; 323(24):2493-2502. doi:10.1001/jama.2020.8630.

8. Wu Cl, Postema PG, Arbelo E, Behr ER, Bezzina CR, Napolitano C, et al. (SARS-CoV-2, COVID-19 and inherited arrhythmia syndromes. Heart Rhythm2020; 17(9):1456-62. doi: 10.1016/j.hrthm.2020.03.024.

9. Ohara H, Nakamura $Y$, Watanabe $Y$, Cao $X$, Yamazaki $Y$, Izumi-Nakaseko $\mathrm{H}$, et al. Azithromycin can prolong QT interval and suppress ventricular contraction, but will not induce torsade de pointes. Cardiovascular toxicology; 15(3): 232-40. doi: 10.1007/s12012-014-9289-4.

10. Drew BJ, Ackerman MJ, Funk M, Gibler WB, Kligfield P, Menon V, et al. Prevention of torsade de pointes in hospital settings: A scientific statement from the American heart association and the American college of cardiology foundation. Circulation 2010; 121(8):1047-60. doi: 10.1016/j.jacc.2010.01.001.

11. Bessière $F$, Roccia $H$, Delinière $A$, Charrière $R$, Chevalier $P$, Argaud, L, et al. Assessment of QT intervals in a case series of patients with coronavirus disease 2019 (COVID-19) infection treated with hydroxychloroquine alone or in combination with azithromycin in an intensive care unit. JAMA Cardiolo 2020; 5(9):1067-9. doi: 10.1001/jamacardio. 2020.1787.

12. Mercuro NJ, Yen CF, Shim DJ, Maher TR, McCoy CM, Zimetbaum PJ, et al. Risk of QT interval prolongation associated with use of hydroxychloroquine with or without concomitant azithromycin among hospitalized patients testing positive for coronavirus disease 2019 (COVID-19). JAMA Cardiology 2020; 5(9):1036-41. doi: 10.1001/jamacardio.2020.1834.

13. Mahalingam D, Mita M, Sarantopoulos J, Wood L, Amaravadi RK, Davis LE, et al. Combined autophagy and HDAC inhibition: A phase I safety, tolerability, pharmacokinetic, and pharmacodynamic analysis of hydroxychloroquine in combination with the HDAC inhibitor vorinostat in patients with advanced solid tumors. Autophagy 2014; 10(8): 1403-14. doi: 10.4161/auto.29231.

14. Traebert $M$, Dumotier $B$, Meister $L$, Hoffmann $P$, DominguezEstevez M, Suter W. Inhibition of hERG K+ currents by antimalarial drugs in stably transfected HEK293 cells. Eur J Pharmacol 2004; 484(1):41-8. doi: 10.1016/ j.ejphar.2003. 11.003.

15. Demazière J, Fourcade JM, Busseuil CT, Adeleine P, Meyer SM, Saïssy JM. The hazards of chloroquine self prescription 
in West Africa. J Toxicol Clin Toxicol 1995; 33(4): 369-70. doi: 10.3109/15563659509028925.

16. Lakkireddy DR, Chung MK, Gopinathannair R, Patton KK, Gluckman TJ, Turagam M, et al. Guidance for cardiac electrophysiology during the COVID-19 pandemic from the heart rhythm society COVID-19 task force; electrophysiology section of the American college of cardiology; and the electrocardiography and arrhythmias committee of the council on clinical cardiology, American heart association. Circulation 2020; 141(21):e823-31. doi: 10.1161/CIRCULATIONAHA. 120.047063.

17. Mazzanti A, Briani M, Kukavica D, Bulian F, Marelli S, Tran- cuccio A, et al. Association of Hydroxychloroquine with QTC Interval in patients with COVID-19. Circulation 2020; doi: 10.1161/CIRCULATIONAHA.120.048476.

18. Chorin E, Wadhwani L, Magnani S, Dai M, Shulman E, Nadeau-Routhier C, et al. QT interval prolongatiPon and torsade De pointes in patients with COVID-19 treated with Hydroxychloroquine/azithromycin. Heart rhythm 2020; 17(9):1425-33. doi: 10.1016/j.hrthm.2020.05.014.

19. Kuate LM, Ngatchou W, Temgoua MN, Kouanfack C, Lemogoum D, Tochie JN, et al. Electrocardiographic safety of daily Hydroxychloroquine $400 \mathrm{mg}$ plus Azithromycin $250 \mathrm{mg}$ as an ambulatory treatment for COVID-19 patients in Cameroon. Medrxiv. 